\section{Transrectal ultrasound-guided prostate biopsy versus combined magnetic resonance imaging-ultrasound fusion and systematic biopsy for prostate cancer detection in routine clinical practice}

Jae Heung Bae, See Hyung Kim

Department of Radiology, School of Medicine, Kyungpook National University, Kyungpook National University Hospital, Daegu, Korea

Purpose: The goal of this study was to retrospectively compare systematic ultrasound-guided prostate biopsy (US-PB) and multiparametric magnetic resonance imaging-ultrasound fusion prostate biopsy (MRI-PB) in men undergoing primary or repeated biopsies.

Methods: A population of 2,200 patients with a prostate-specific antigen (PSA) level $>4.0 \mathrm{ng} /$ $\mathrm{dL}$ and/or an abnormal rectal examination was divided into two groups. All patients underwent US-PB $(n=1,021)$ or MRI-PB $(n=1,179)$ between April 2015 and April 2019. Population demographics, including age, PSA level, digital rectal examination results, prostate volume, number of previous negative biopsies, Prostate Imaging Reporting and Data System (PI-RADS) version 2 (V2) score, and biopsy results, were acquired and compared with respect to these variables. Univariate regression analysis of the risk factors for a higher Gleason score (GS) was performed.

Results: The cancer detection rate (CDR) was $23.8 \%(243$ of 1,021) in the US-PB group and $31.3 \%$ (399 of 1,179$)$ in the MRI-PB group. Of those, 225 patients $(22.0 \%)$ in the US-PB group and 374 patients (31.7\%) in the MRI-PB group had clinically significant prostate cancer (csPCa). The patients with csPCa in the MRI-PB group included 10 (40\%), 50 (62.5\%), 184 (94.8\%), and 32 (94.1\%) patients with PI-RADS V2 scores of 2, 3, 4, and 5, respectively. Of the patients with cSPCa, $155(91.7 \%)$ in the US-PB group were diagnosed on the basis of the primary biopsy, compared to $308(94.4 \%)$ in the MRI-PB group. We found the PI-RADS V2 score to be the best predictor of a higher GS.

Conclusion: MRI-PB showed a high CDR for csPCa. MRI-PB could be a reasonable approach in patients with high PI-RADS V2 scores at primary biopsy.

Keywords: Prostatic cancer; Image-guided biopsy; Ultrasonography; Magnetic resonance imaging

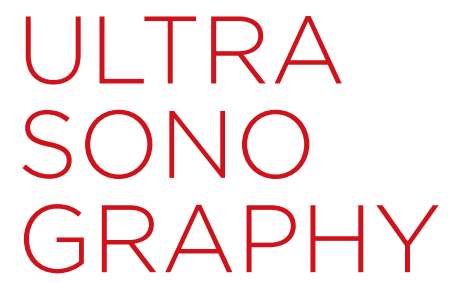

\section{ORIGINAL ARTICLE}

https://doi.org/10.14366/usg. 19036 pISSN: 2288-5919 • elSSN: 2288-5943

Ultrasonography 2020;39:137-143

Received: June 22, 2019

Revised: November 15, 2019

Accepted: November 26, 2019

Correspondence to:

See Hyung Kim, MD, PhD, Department of Radiology, School of Medicine, Kyungpook National University, Kyungpook National University Hospital, 130 Dongdeok-ro, Jung-gu, Daegu 41944, Korea

Tel. +82-53-200-5390

Fax. +82-53-422-2677

E-mail: kimseehyung72@outlook.kr

This is an Open Access article distributed under the terms of the Creative Commons Attribution NonCommercial License (http://creativecommons.org/ licenses/by-nc/4.0/) which permits unrestricted noncommercial use, distribution, and reproduction in any medium, provided the original work is properly cited.

Copyright (C) 2020 Korean Society of Ultrasound in Medicine (KSUM)

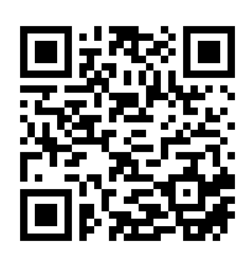

How to cite this article:

Bae JH, Kim SH. Transrectal ultrasoundguided prostate biopsy versus combined magnetic resonance imaging-ultrasound fusion and systematic biopsy for prostate cancer detection in routine clinical practice. Ultrasonography. 2020 Apr;39(2):137-143. 


\section{Introduction}

The introduction of prostate-specific antigen (PSA) screening into routine clinical practice several decades ago has led to more biopsies and increased detection of prostate cancer [1]. Traditionally, prostate cancer is detected with PSA screening, digital rectal examination (DRE), and systematic ultrasound-guided prostate biopsy (US-PB) [2-4]. This approach is associated with the increased detection of earlier-stage prostate cancers with lower Gleason scores. The use of US-PB has been criticized for leading to the overdiagnosis of lowrisk prostate cancer and the underdiagnosis of clinically significant prostate cancer (csPCa) [5-7].

More recently, multiparametric magnetic resonance imaging (mpMRI) has become an important method for the detection of localized prostate cancer. Ultrasound combined with mpMRI in a fusion technique for prostate biopsy (MRI-PB), which merges previously captured mpMRI images with live transrectal ultrasound images, is a promising method for the detection of csPCa [6-9]. MRI-PB has proven to be effective for accurately targeting lesions in prostate areas that are traditionally missed or hard to reach with US-PB, such as the anterior prostate, transition zone, distal apical region, and posterior subcapsular region. In addition, many studies have shown that MRI-PB is superior to US-PB in terms of adverse event-associated morbidity $[8,9]$. Although professional guidelines currently recommend MRI-PB, its implementation as a primary methodology is still under assessment. Most patients still undergo US-PB, even in centers where both techniques are available.

The purpose of this study was to retrospectively assess US-PB and MRI-PB in men undergoing primary and repeated biopsies to compare the cancer detection rate (CDR) of csPCa. The relationship between the number of previous biopsies undergone by the patient and the CDR was also analyzed. Additionally, we assessed the correlation between the CDR and the results of the Prostate Imaging Reporting and Data System (PI-RADS) version 2 (V2) scoring system.

\section{Materials and Methods}

\section{Patient Population}

This was a single-center study based on routine clinical practice. The examined patients included those at their first prostate check and those with previous negative prostate biopsies. Patients' risk of prostate cancer was assessed by PSA level and/or DRE. The study protocol was approved by our institutional board review committee, and informed consent was obtained from all patients. Between April 2015 and April 2019 (the study period), a total of 2,500 patients at our urology outpatient clinic with a PSA level $>4.0 \mathrm{ng} / \mathrm{dL}$ and/or abnormal DRE underwent US-PB or MRI-PB. The patient selection process is shown in Fig. 1. Patients presenting with metastatic disease and symptoms of locally advanced disease were excluded. Patients under active surveillance who had already been diagnosed with low-grade cancer were also excluded. There were 2,200 patients in the final study population. We divided the population into two non-randomized groups: 1,021 patients underwent standard, laterally directed, random US-PB, while the remaining 1,179 patients underwent MRI-PB. A total of 485 men with no suspicious target lesion shown on mpMRI underwent US-PB.

\section{mpMRI}

Magnetic resonance imaging (MRI) was performed using the following 3-T magnetic resonance imagers: Skyra (Siemens Healthineers, Erlangen, Germany), Achieva and Ingenia CX (Philips Medical System, Best, Netherlands), and the 750W Architect (GE Medical Systems, Milwaukee, WI, USA) with a multichannel phased array body surface coil. An intramuscular injection of $1 \mathrm{mg}$ of glucagon was administered to suppress bowel peristalsis. The MRI protocol included T2-weighted fast spin-echo sequences (repetition time [TR] , 4,500 msec; echo time [TE] 110 msec; thickness, 3 mm; and matrix, $352 \times 352)$ in the axial, sagittal, and coronal planes, as well as diffusion-weighted sequences (slice thickness, $3 \mathrm{~mm}$; TR, 3,100 msec; TE, $102 \mathrm{msec}$; and exponential b-values of 0,500, and $1,000 \mathrm{sec}\left(\mathrm{mm}^{2}\right)$. Dynamic contrast-enhanced sequences were obtained with a gradient echo T1-weighted sequence in the axial plane (TR, 3 msec; TE, 2 msec; thickness, $3 \mathrm{~mm}$; time resolution, 12 sections/3 sec; and matrix, 320×192).

\section{Prostate Biopsy}

The decision to assess the patient using US-PB or MRI-PB was made by the patient's urologist according to the clinical situation or cost considerations. US-PB was performed using a transrectal ultrasound system (EPIQ 7, Philips Healthcare, Best, Netherlands), and MRIPB was performed using an office-based platform (UroNav, Philips/ In Vivo Corp., Gainesville, FL, USA). MRI-PB involves a series of steps. The prostate gland is first outlined through axial T2-weighted images to set three key points: the base, apex, and rear portions of the prostate. This process allows the software to create a threedimensional model of the gland on which the operator can mark areas that represent target lesions previously identified by mpMRI. Once this is completed, the fusion process between MRI and ultrasonography (US) images takes place.

Patients who had a suspicious target lesion identified on mpMRI underwent target and systematic biopsy in the same session. Lesions identified on mpMRI were interpreted using the PI-RADS V2 scoring system (1, clinically significant cancer highly unlikely; 2, clinically significant cancer unlikely; 3 , clinically significant cancer equivocal; 4, 


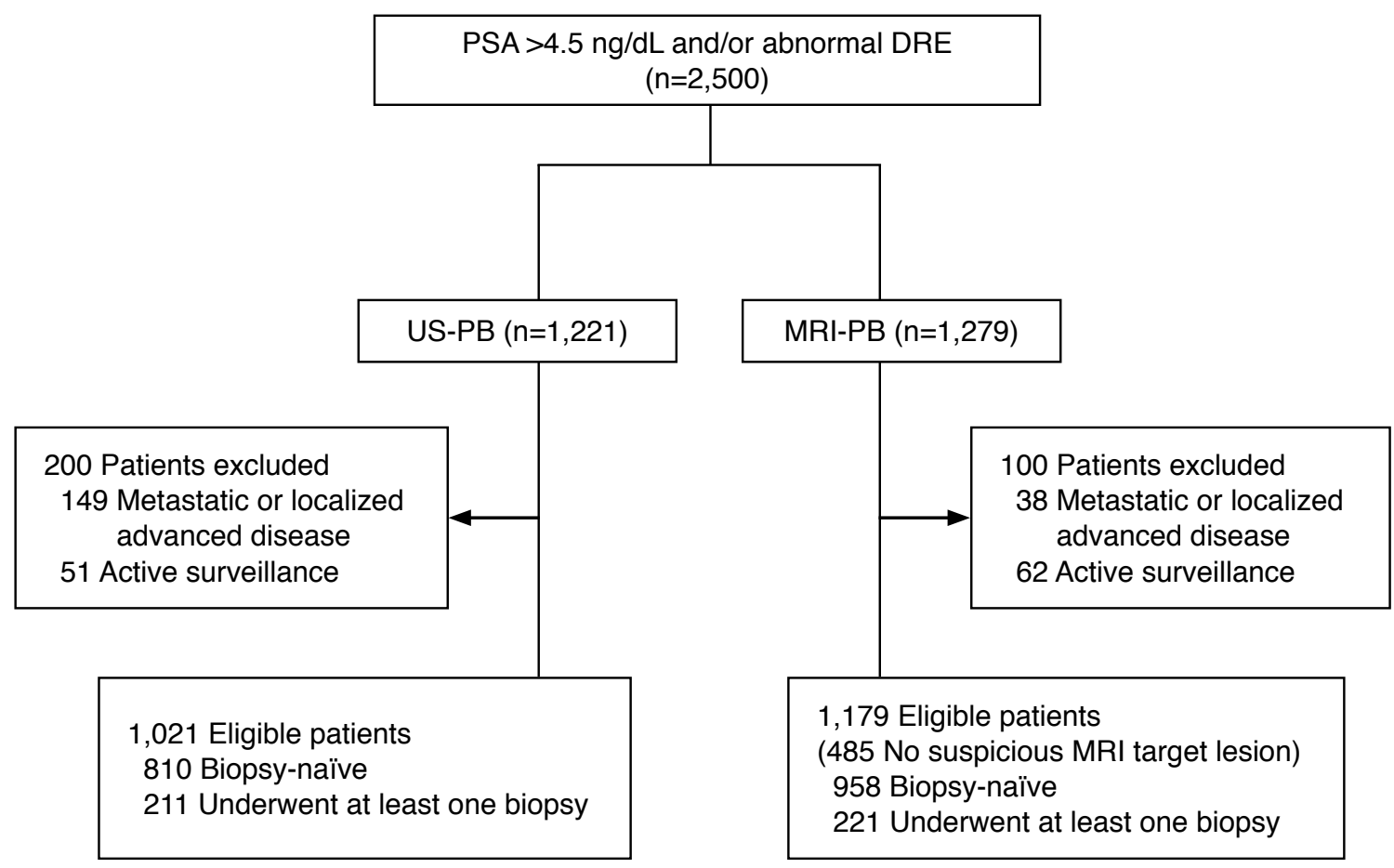

Fig. 1. Flowchart of patient selection process. PSA, prostate-specific antigen; DRE, digital rectal examination; US-PB, systematic ultrasoundguided prostate biopsy; MRI-PB, multiparametric magnetic resonance imaging (mpMRI)-ultrasound fusion prostate biopsy.

clinically significant cancer likely; and 5, clinically significant cancer highly likely). Lesions with scores $\geq 2$ were defined as suspicious target lesions. In cases with a single lesion, two targeted core biopsy specimens were taken, and in cases with two suspicious target lesions, one core biopsy from each lesion was obtained. If more than two target lesions were identified on mpMRI, one core biopsy was obtained from each of the two lesions with the highest PI-RADS V2 scores. Review of the mpMRI images was performed by one of three radiologists with 8-12 years of experience in using prostate mpMRI to identify suspicious target lesions in the prostate gland.

An $18 \times 25-\mathrm{cm}$ spring-loaded core needle biopsy instrument (TSKCore) was used to obtain biopsy specimens in both groups. For each patient, US-PB and MRI-PB were performed by the same faculty radiologist with expertise in prostate biopsy.

\section{Histopathology}

Each biopsy core was labeled, processed, and examined separately by an experienced uropathologist who was blinded to the mpMRI results. The criteria for csPCa were defined according to Epstein et al. [10] and included either a biopsy specimen with Gleason score (GS) $>6$ and $50 \%$ involvement of prostate cancer per core or prostate cancer detected in more than two cores.

\section{Statistical Analysis}

To compare variables between the two patient groups, the 2-tailed $t$ test was performed with a $5 \%$ type I error. The Welch 2-sample $t$ test was used to verify differences in the groups. Univariate regression analysis of the risk factors for an elevated GS (age, PSA, DRE, prostatic volume measured by transrectal ultrasound biopsy [with the ellipsoid formula=anteroposterior diameter $\times$ transverse diameter $\times$ sagittal diameter $\times \pi / 6]$, number of previous negative US-guided biopsies, and PI-RADS V2 score for target lesions) was performed, and a P-value less than 0.05 was considered to indicate statistical significance. Statistical analysis was performed using SAS version 9.2 (SAS Institute Inc., Cary, NC, USA).

\section{Results}

We stratified the total study population (2,200 patients) by PIRADS V2 score and by the number of previous biopsies. There were 152 patients with a PI-RADS V2 score of 2, 300 patients with a score of 3, 205 patients with a score of 4, and 37 patients with a score of 5 . In the US-PB group, there were 810 patients at primary biopsy, 111 patients with one previous negative biopsy, 66 patients with two previous negative biopsies, and 34 patients with three or more previous negative biopsies. In the MRI-PB group, there were 
Table 1. Characteristics of the population

\begin{tabular}{|c|c|c|}
\hline Variable & US-PB $(n=1,021)$ & MRI-PB $(n=1,179)$ \\
\hline Age (yr) & $75.2(55.5-85.7)$ & $69.3(56.7-90.5)$ \\
\hline PSA (ng/dL) & $10.7(5.0-20.4)$ & $7.2(5.6-24.8)$ \\
\hline Prostate volume $(\mathrm{mL})$ & $41.5(26.5-62.4)$ & $55.7(30.6-56.9)$ \\
\hline \multicolumn{3}{|l|}{ DRE } \\
\hline Normal & 532 & 500 \\
\hline Abnormal & 489 & 679 \\
\hline No. of target lesions per patient & NA & $2(1-3)$ \\
\hline \multicolumn{3}{|l|}{ Cancer core length (mm) } \\
\hline Systematic biopsy & $8.5(6.5-10.7)$ & $9.2(6.3-11.3)$ \\
\hline Target biopsy & NA & $12.0(9.2-12.9)$ \\
\hline
\end{tabular}

958 patients at primary biopsy, 132 patients with one previous negative biopsy, 61 patients with two previous negative biopsies, and 28 patients with three or more previous negative biopsies. The characteristics of the population are summarized in Table 1.

The overall CDR was 23.8\% (243 patients) in the US-PB group and 31.3\% (399 patients) in the MRI-PB group. csPCa was detected in 225 patients $(22.0 \%)$ in the US-PB group and in 374 patients $(31.7 \%)$ in the MRI-PB group. Among patients in the MRI-PB group without a suspicious target lesion, 66 patients (13.6\%) were diagnosed by US-PB only, and csPCa was detected in 60 of these patients (12.3\%). With regard to the PI-RADS V2 score, the CDR was $7.6 \%$ (25 patients), 24.0\% (80 patients), 58.2\% (194 patients), and $10.2 \%$ (34 patients) among those with scores of 2, 3, 4, and 5 , respectively.

The CDR by number of previous biopsies was $68.7 \%$ (167 patients) in US-PB patients and $83.4 \%$ (278 patients) in MRI-PB patients at primary biopsy, $17.6 \%$ (43 patients) in US-PB patients and $13.2 \%$ (44 patients) in MRI-PB patients with one previous negative biopsy, $11.1 \%$ (27 patients) in US-PB patients and $2.7 \%$ (9 patients) in MRI-PB patients with two previous negative biopsies, and $2.6 \%$ (6 patients) in US-PB patients and $0.7 \%$ (2 patients) in MRI-PB patients with three or more previous negative biopsies. Among patients in the MRI-PB group without a suspicious target lesion, the overall CDR was $72.7 \%$ (48 patients) at primary biopsy, $18.1 \%$ (12 patients) in patients with one previous negative biopsy, $7.5 \%$ (5 patients) in patients with two previous negative biopsies, and $1.7 \%$ (1 patient) among those with three or more previous negative biopsies. The distribution of GS at biopsy in relation to PIRADS V2 score and the number of previous biopsies is specified in Table 2.
Patients with csPCa in the MRI-PB group had the following distribution of PI-RADS V2 scores: 10 patients (40\%) with a score of 2, 50 patients $(62.5 \%)$ with a score of 3,184 patients $(94.8 \%)$ with a score of 4 , and 32 patients (94.1\%) with a score of $5(P<0.05)$.

Furthermore, 155 patients with csPCa $(91.7 \%)$ in the USPB group and 308 patients $(94.4 \%)$ in the MRI-PB group were diagnosed at primary biopsy $(P<0.05)$. Additionally, there were 263 patients $(94.6 \%)$ in the MRI-PB group and 45 patients $(93.7 \%)$ in the MRI-PB group without a suspicious target lesion.

In the univariate analysis, PI-RADS V2 score was the best predictor of an elevated GS and showed a significant correlation (Pearson's coefficient, $P<0.05$ ) ( $B$ for a PI-RADS score of $4,0.82$; $\beta$ for a PIRADS score of 5,1$)$.

\section{Discussion}

The management of prostate biopsy in men with clinical suspicion of prostate cancer has changed in recent years. Over the past 10 years, the widespread use of PSA as a screening test has led to an increased number of prostate cancer diagnoses, resulting in the possibility of overtreatment. In the past few years, research has focused on developing more accurate imaging techniques to overcome the practical limitations of PSA screening [11-13]. To implement proper treatment, extensive efforts have been made to identify csPCa. Several recent studies showed that mpMRI combined with MRI-PB is a promising method for the detection of prostate cancer, particularly for csPCa. Despite these promising results, European Association of Urology guidelines recommend mpMRI only for patients with suspected prostate cancer (grade B) who have a previous negative prostate biopsy [14-16].

This study demonstrates that MRI-PB has the potential to improve the detection of prostate cancer and clinical outcomes by accelerating the understanding of how MRI-PB can be incorporated successfully into routine clinical practice. Across the two groups (US-PB vs. MRI-PB with a suspicious target lesion), the CDR was $23.8 \%$ and $47.9 \%$, respectively, and csPCa was detected at similar rates (22.0\% in the US-PB group and $45.1 \%$ in the MRI-PB group). $A$ high rate of $\operatorname{cSPCa}$ was detected in the MRI-PB group with a suspicious target lesion. We believe that mpMRI followed by MRI-PB has meaningful advantages in terms of overall CDR, particularly with regard to the $\operatorname{csPCa}$ detection rate.

As expected, the two groups were not comparable and may have not had the same risk of prostate cancer. When both biopsy techniques are available, younger patients with a previous history of negative biopsy, a higher PSA, and larger prostate volume are more likely to be advised to undergo MRI-PB. Specifically, in the present study, 485 patients without a suspicious target lesion were included 
Table 2. Cancer detection rate and Gleason pattern in correlation to the number of previous biopsies and PI-RADS V2 score

\begin{tabular}{|c|c|c|c|c|c|}
\hline Biopsy result & No. of patients & Cancer detection rate & Gleason score 6 & Gleason score 7 & Gleason score 8-10 \\
\hline US-PB & 1,021 & $243(23.8)$ & $18(7.5)$ & $162(66.6)$ & $63(25.9)$ \\
\hline MRI-PB & 694 & $333(47.9)$ & $20(6.1)$ & $173(51.9)$ & $140(42.0)$ \\
\hline MRI-PB (no target lesion) & 485 & $66(13.6)$ & $5(7.7)$ & $37(56.0)$ & $24(36.3)$ \\
\hline \multicolumn{6}{|c|}{$\begin{array}{l}\text { Cancer detection rate in relation to } \\
\text { number of previous biopsies }\end{array}$} \\
\hline \multicolumn{6}{|l|}{ US-PB } \\
\hline Primary biopsy & 810 & $167(68.7)$ & $12(66.7)$ & $108(66.6)$ & $47(74.6)$ \\
\hline 1 & 111 & $43(17.6)$ & $2(11.1)$ & $31(19.1)$ & $10(15.8)$ \\
\hline 2 & 66 & $27(11.1)$ & $2(11.1)$ & $20(12.3)$ & $5(7.9)$ \\
\hline 3 or more & 34 & $6(2.6)$ & $2(11.1)$ & $3(2.0)$ & $1(1.7)$ \\
\hline \multicolumn{6}{|l|}{ MRI-PB } \\
\hline Primary biopsy & 632 & $278(83.4)$ & $15(75.0)$ & $143(82.6)$ & $120(85.7)$ \\
\hline 1 & 89 & $44(13.2)$ & $5(25.0)$ & $23(13.2)$ & $16(11.4)$ \\
\hline 2 & 39 & $9(2.7)$ & 0 & $5(2.8)$ & $4(2.9)$ \\
\hline 3 or more & 18 & $2(0.7)$ & 0 & $2(1.4)$ & 0 \\
\hline \multicolumn{6}{|l|}{ MRI-PB (no target lesion) } \\
\hline Primary biopsy & 326 & $48(72.7)$ & $3(60.0)$ & $25(67.5)$ & $20(83.3)$ \\
\hline 1 & 43 & $12(18.1)$ & $1(20.0)$ & $8(21.6)$ & $3(12.5)$ \\
\hline 2 & 22 & $5(7.5)$ & $1(20.0)$ & $3(8.1)$ & $1(4.2)$ \\
\hline 3 or more & 10 & $1(1.7)$ & 0 & $1(2.8)$ & 0 \\
\hline \multicolumn{6}{|c|}{$\begin{array}{l}\text { Cancer detection rate in relation to } \\
\text { PI-RADS V2 score }\end{array}$} \\
\hline 2 & 152 & $25(7.6)$ & $15(60.0)$ & $8(32.0)$ & $2(8.0)$ \\
\hline 3 & 300 & $80(24.0)$ & $30(37.5)$ & $37(46.2)$ & $13(16.3)$ \\
\hline 4 & 205 & $194(58.2)$ & $10(5.2)$ & $79(40.7)$ & $105(54.1)$ \\
\hline 5 & 37 & $34(10.2)$ & $2(8.8)$ & $12(32.4)$ & $20(58.8)$ \\
\hline
\end{tabular}

Values are presented as number (\%).

PI-RADS V2, Prostate Imaging Reporting and Data System version 2; US-PB, systematic ultrasound-guided prostate biopsy; MRI-PB, multiparametric magnetic resonance imaging-ultrasound fusion prostate biopsy.

in the MRI-PB group and underwent only US-PB. These patients were analyzed separately to prevent induced selection bias, as they had a similar risk of prostate cancer as patients in the US-PB group.

The MRI-PB - only group can be considered a very select population, as these patients were previously screened by mpMRI and only those with a PI-RADS V2 score were included. The mean PIRADS V2 score in the MRI-PB group was 3.15. For a homogeneous population, such as that observed in clinical practice, our data appear to confirm the previously reported results that a higher PIRADS V2 score correlates with the likelihood of detected csPCa [1517].

MRI-PB was mainly performed in patients who had at least one previous negative biopsy and whom we suspected to have an anteriorly located csPCa or cancer focus inside a large volume of benign prostatic hyperplasia. Our results showed that the noteworthy improvement in CDR for the MRI-PB group may not apply to patients with $>2$ previous negative biopsies. Moreover, the results showed no difference in detection rate in patients with $>2$ previous negative biopsies between the US-PB group and the MRIPB group. Similar to our results, previous studies have concluded that the utility of MRI-PB is limited both in biopsy-naivve patients and those with one previous negative biopsy [17-21].

Limitations of this study include the potential for selection bias, given that it was a single-center study with a limited population; this raises the question of whether the results are reproducible at other centers. However, we believe that the consecutive nature of our cohort minimizes the possibility of bias to an extent, as patients were largely referred based on community screening practices. The mpMRI interpretation was performed by experienced radiologists, and it is well known that radiologists can have differing 
interpretations of PI-RADS V2 scores. However, the increasing popularity of mpMRI and improved consensus between radiologists may lead to greater uniformity of image interpretation in the future. Additionally, our conclusions regarding cancer detection were based purely on biopsy results and were not validated by prostatectomy findings. Disease risk in our study was defined based on risk stratification methods derived from biopsy results. Furthermore, our study is limited by the lack of systematic histological confirmation of the information obtained by prostate biopsy (most notably in the case of negative biopsies), with false-negative results being impossible to assess. Despite this, we believe these methods offer the best means to assess risk in the context of biopsies.

In conclusion, MRI-PB showed a high CDR for CSPCa. Moreover, MRI-PB may be a reasonable approach for patients with high PIRADS V2 scores at primary biopsy.

ORCID: Jae Heung Bae: https://orcid.org/0000-0002-4123-4693; See Hyung Kim: https://orcid.org/0000-0002-3268-3091

\section{Author Contributions}

Conceptualization: Kim SH. Data acquisition: Bae JH, Kim SH. Data analysis or interpretation: Bae JH, Kim SH. Drafting of the manuscript: Bae JH, Kim SH. Critical revision of the manuscript: Kim SH. Approval of the final version of the manuscript: all authors.

\section{Conflict of Interest}

No potential conflict of interest relevant to this article was reported.

\section{References}

1. Mouraviev V, Villers A, Bostwick DG, Wheeler TM, Montironi $R$, Polascik TJ. Understanding the pathological features of focality, grade and tumour volume of early-stage prostate cancer as a foundation for parenchyma-sparing prostate cancer therapies: active surveillance and focal targeted therapy. BJU Int 2011;108:1074-1085.

2. Stamey TA, McNeal JE, Yemoto CM, Sigal BM, Johnstone IM. Biological determinants of cancer progression in men with prostate cancer. JAMA 1999;281:1395-1400.

3. Shukla-Dave A, Hricak H, Akin O, Yu C, Zakian KL, Udo K, et al. Preoperative nomograms incorporating magnetic resonance imaging and spectroscopy for prediction of insignificant prostate cancer. BJU Int 2012;109:1315-1322.

4. Sciarra A, Barentsz J, Bjartell A, Eastham J, Hricak H, Panebianco $V$, et al. Advances in magnetic resonance imaging: how they are changing the management of prostate cancer. Eur Urol 2011;59:962-977.

5. Hoeks CM, Barentsz JO, Hambrock T, Yakar D, Somford DM,
Heijmink SW, et al. Prostate cancer: multiparametric MR imaging for detection, localization, and staging. Radiology 2011;261:46-66.

6. Puech P, Rouviere $\mathrm{O}$, Renard-Penna R, Villers $A$, Devos $P$, Colombel $M$, et al. Prostate cancer diagnosis: multiparametric MR-targeted biopsy with cognitive and transrectal US-MR fusion guidance versus systematic biopsy: prospective multicenter study. Radiology 2013;268:461-469.

7. Pinto PA, Chung PH, Rastinehad AR, Baccala AA Jr, Kruecker J, Benjamin $C J$, et al. Magnetic resonance imaging/ultrasound fusion guided prostate biopsy improves cancer detection following transrectal ultrasound biopsy and correlates with multiparametric magnetic resonance imaging. J Urol 2011;186:1281-1285.

8. Moore CM, Robertson NL, Arsanious N, Middleton T, Villers A, Klotz $\mathrm{L}$, et al. Image-guided prostate biopsy using magnetic resonance imaging-derived targets: a systematic review. Eur Urol 2013;63:125-140.

9. Siddiqui MM, Rais-Bahrami S, Truong H, Stamatakis L, Vourganti S, Nix J, et al. Magnetic resonance imaging/ultrasound-fusion biopsy significantly upgrades prostate cancer versus systematic 12-core transrectal ultrasound biopsy. Eur Urol 2013;64:713-719.

10. Epstein JI, Egevad L, Amin MB, Delahunt B, Srigley JR, Humphrey PA, et al. The 2014 International Society of Urological Pathology (ISUP) Consensus Conference on Gleason Grading of Prostatic Carcinoma: definition of grading patterns and proposal for a new grading system. Am J Surg Pathol 2016;40:244-252.

11. Kasivisvanathan $\mathrm{V}$, Dufour $\mathrm{R}$, Moore CM, Ahmed HU, Abd-Alazeez $M$, Charman SC, et al. Transperineal magnetic resonance image targeted prostate biopsy versus transperineal template prostate biopsy in the detection of clinically significant prostate cancer. J Urol 2013;189:860-866.

12. Radtke JP, Kuru TH, Bonekamp D, Freitag MT, Wolf MB, Alt CD, et al. Further reduction of disqualification rates by additional MRItargeted biopsy with transperineal saturation biopsy compared with standard 12-core systematic biopsies for the selection of prostate cancer patients for active surveillance. Prostate Cancer Prostatic Dis 2016;19:283-291.

13. Mozer P, Roupret M, Le Cossec C, Granger B, Comperat E, de Gorski $A$, et al. First round of targeted biopsies using magnetic resonance imaging/ultrasonography fusion compared with conventional transrectal ultrasonography-guided biopsies for the diagnosis of localised prostate cancer. BJU Int 2015;115:50-57.

14. Sonn GA, Chang E, Natarajan S, Margolis DJ, Macairan M, Lieu P, et al. Value of targeted prostate biopsy using magnetic resonanceultrasound fusion in men with prior negative biopsy and elevated prostate-specific antigen. Eur Urol 2014;65:809-815.

15. Hansen NL, Koo BC, Gallagher FA, Warren AY, Doble A, Gnanapragasam V, et al. Comparison of initial and tertiary centre second opinion reads of multiparametric magnetic resonance imaging of the prostate prior to repeat biopsy. Eur Radiol 
2017;27:2259-2266.

16. Schroder FH, Hugosson J, Roobol MJ, Tammela TL, Ciatto S, Nelen $\mathrm{V}$, et al. Prostate-cancer mortality at 11 years of follow-up. $N$ Engl J Med 2012;366:981-990.

17. Sciarra A, Panebianco V, Ciccariello M, Salciccia S, Cattarino S, Lisi $D$, et al. Value of magnetic resonance spectroscopy imaging and dynamic contrast-enhanced imaging for detecting prostate cancer foci in men with prior negative biopsy. Clin Cancer Res 2010;16:1875-1883.

18. Hong CW, Rais-Bahrami S, Walton-Diaz A, Shakir N, Su D, George $A K$, et al. Comparison of magnetic resonance imaging and ultrasound (MRI-US) fusion-guided prostate biopsies obtained from axial and sagittal approaches. BJU Int 2015;115:772-779.
19. Rais-Bahrami S, Siddiqui MM, Turkbey B, Stamatakis L, Logan J, Hoang AN, et al. Utility of multiparametric magnetic resonance imaging suspicion levels for detecting prostate cancer. J Urol 2013;190:1721-1727.

20. Valerio M, Donaldson I, Emberton M, Ehdaie B, Hadaschik BA, Marks $L S$, et al. Detection of clinically significant prostate cancer using magnetic resonance imaging-ultrasound fusion targeted biopsy: a systematic review. Eur Urol 2015;68:8-19.

21. Costa DN, Lotan Y, Rofsky NM, Roehrborn C, Liu A, Hornberger B, et al. Assessment of prospectively assigned Likert scores for targeted magnetic resonance imaging-transrectal ultrasound fusion biopsies in patients with suspected prostate cancer. J Urol 2016;195:80-87. 